\title{
A Unified Analysis of Conditional Imperatives
}

\author{
Stefan Kaufmann \\ Northwestern University
}

\author{
Magdalena Schwager \\ University of Göttingen
}

For the purposes of this paper, conditional imperatives (henceforth CIs) are indicative conditionals whose consequent has the grammatical form of an imperative, illustrated in (1).
a. If you are at an intersection, turn right!
b. If you reach an intersection, turn right!

The main goal of this paper is to develop a unified semantic analysis of CIs, one which adequately captures their similarities to (unconditional) imperatives on the one hand and (unimperative) conditionals, on the other. Both of those latter phenomena have been explored extensively and, differences over theoretical framework and formal implementation notwithstanding, are comparatively well-understood. In tackling CIs, then, the first question to ask is whether or to what extent their semantic behavior can be captured by simply combining an analysis of imperatives with one of conditionals. We show in this paper that the answer is affirmative in principle, but that CIs also raise a number of specific challenges which in turn inform the analysis of imperatives and conditionals in general.

\section{Preliminaries}

Before we begin to present our unified account, the first question is which analyses for conditionals and imperatives to build it on. In particular, conditionals are known as a class of constructions rather than a uniform phenomenon (cf. Bhatt and Pancheva, 2006). Besides hypothetical conditionals as in (2), we find at least relevance and factual conditionals.

(2) a. If she is smart, she accepted the offer.

b. If she gets the offer, she will accept it.
a. If you are thirsty, there is a beer in the frige.
[relevance conditional]
b. If you like him, you should help him.
[factual conditional]

Broadly speaking, hypothetical conditionals like those in (2) assert that a certain relationship holds between the truth of the antecedent and that of the consequent. In contrast, relevance and factual conditionals are often grouped together as speech act conditionals as they express a dependence between the truth of the antecedent

We thank the audience at SALT 19 for comments and discussions. 
and the relevance or felicity of the speech act performed with the consequent. Even in case of an assertive consequent, the truth of the consequent is only indirectly involved inasmuch as it figures at the speech-act level.

One might not expect CIs to ever constitute hypothetical conditionals. It is often argued that the question of "truth" is beside the point when it comes to imperatives, and that they should instead be analyzed in terms of the felicity and successful execution of non-constative speech acts. However, Schwager (2006a) shows that according to standard tests (cf. Bhatt and Pancheva, 2006), all three types of conditionals can be exemplified by CIs. In particular, modification with only and binding from the consequent into the antecedent occur with hypothetical conditionals only. Under these criteria, the CIs in (4) and (5) are hypothetical conditionals.

(4) a. Turn right only if you are at an intersection.

b. Call a doctor only if you are sick.

a. If you really like $\mathrm{it}_{i}$, [a donkey $]_{i}$ will be grateful.

b. If it $_{i}$ is tired, let [a donkey $]_{i}$ rest.

In contrast, the CIs in (6) arguably constitute speech act conditionals.

$$
\begin{aligned}
& \text { a. If I may give you some advice, don't go. } \\
& \text { b. If you are so smart, then do it yourself. }
\end{aligned}
$$

[relevance $\mathrm{CI}$ ]

[factual CI]

The upshot is that CIs do not belong to a single class of conditionals, and a truly comprehensive unified account must treat each of the subclasses on a par with the corresponding non-imperative conditionals. In this paper, however, we stop short of attempting such broad coverage, focusing instead on hypothetical CIs with the more modest goal of providing an account which unifies them with ordinary hypothetical conditionals. What we need, then, is an analysis of imperatives in terms of truth conditions and a framework for conditionals that is compatible with this analysis.

\section{Formal Background}

While we aim to keep the discussion rather informal throughout most of the paper, we do make reference to a few formal model-theoretic notions. In this section we briefly introduce those tools. We take as given a non-empty set $W$ of possible worlds. Atomic sentences denote subsets of $W$ (i.e., propositions). Our goal is to define the denotations of conditionals, imperatives, and ultimately CIs.

\subsection{Modality and Conditionals}

We follow Kratzer (1981) in analyzing conditionals as modal expressions whose consequent is the prejacent of a(n overt or covert) modal operator and whose antecedent serves to restrict the modal base of that operator. Modal sentences are 
interpreted relative to two parameters $f, g$. Both are conversational backgrounds in Kratzer's terms, i.e., functions from worlds to sets of propositions. The first one determines which worlds form the domain relative to which the modal expressions in question are to be interpreted, much like the accessibility relations famliar in modal logic. For each world $w$, we call the set $\bigcap f(w)$ (the worlds at which all propositions in $f(w)$ are true) the modal base at $w$. The second parameter $g$ is an ordering source inducing, for each world $w$, a pre-order on the set of worlds as defined in (7a). Depending on the way $g$ is interpreted, $u \leq g(w) v$ means that $u$ is less far-fetched than $v$, preferable to $v$, or "better" than $v$ in some other contextually salient respect. We assume for simplicity that $g(w)$ is finite for all $g, w$. Then there is guaranteed to be a set of minimal or "best" worlds for any modal base. We refer to this set as in (7b).

$$
\begin{aligned}
& \text { a. } u \leq g(w) v \text { iff }\{p \in g(w) \mid v \in p\} \subseteq\{p \in g(w) \mid u \in p\} \\
& \text { b. } O(w, f, g):=\{u \in \bigcap f(w) \mid \forall v \in \bigcap f(w)[v \leq g(w) u \rightarrow u \leq g(w) v]\}
\end{aligned}
$$

We add a human necessity modal operator $⿴ 囗 十$ to the language whose interpretation relative to a world $w$, modal base $f$ and ordering source $g$ is defined in (8a). A special case is the simple necessity operator, defined in (8b) as shorthand for human necessity relative to a constant ordering source whose value is the empty set of propositions. In this case the sentence is true iff its prejacent is true at all worlds in the modal base.

a. 䧃 $\varphi$ is true w.r.t. $w, f, g$ iff $\varphi$ is true at all worlds in $O(w, f, g)$.

b. $\square \varphi$ is true w.r.t. $w, f$ iff $⿴ 囗 十$ is true w.r.t. $w, f, \lambda v[\emptyset]$.

As mentioned above, a conditional antecedent acts as a restrictor on the modal base relative to which the consequent is evaluated. Kratzer (1981) assumes that when no overt modal auxiliary is present, the default is an epistemic human-necessity operator. Kaufmann (2005) claims that the default is a simple-necessity operator introduced by tense. Regardless of the source of the modal operator, the interpretation is as defined in (9). Here and below, we schematically add the subscript ' $[\varphi]$ ' to represent a modal operator modified by a conditional antecedent, and we let $\varphi^{\prime}$ stand for the set of worlds at which $\varphi$ is true.

$$
\left.\mathbb{⿴ 囗 十}_{[\varphi]}[\psi] \text { is true w.r.t. } w, f, g \text { iff } ⿴ 囗 十\right) \text { is true w.r.t. } w, \lambda v\left[f(v) \cup\left\{\varphi^{\prime}\right\}\right], g \text {. }
$$

Replacing $f$ with $f^{\prime}=\lambda v\left[f(w) \cup\left\{\varphi^{\prime}\right\}\right]$ has the effect that $\cap f^{\prime}(w)=\bigcap\left[f(w) \cup\left\{\varphi^{\prime}\right\}\right]$ $=[\cap f(w)] \cap \varphi^{\prime}$. Thus the antecedent in (9) does restrict the modal base. It does not follow, however, that $O\left(w, f^{\prime}, g\right)$ and $O(w, f, g)$ stand in any particular relation. For instance, they are disjoint whenever both are non-empty and $\varphi$ is false at all worlds in $O(w, f, g)$. This captures the non-monotonic behavior of conditionals, i.e., the fact that it is possible that ' $⿴ 囗 十 \psi$ ' is true while ' $⿴ 囗 十[\varphi] \psi$ ' is false.

The model as we assume it here does not include a temporal dimension and is therefore ill-suited for the analysis of certain subtle but important details of the interpretation of temporal expressions in conditionals. This is a drawback in the case of CIs as well. For instance, a crucial distinction Kaufmann (2005) makes in his treatment of indicative conditionals is between non-predictive and predictive ones, illustrated in (1) above, repeated here as (10). 
The issue of tense and temporal reference is important, yet many insights about CIs can be gained without going into this topic. We thus decide to leave it aside for the moment.

\subsection{Imperatives}

Imperatives pose problems for any standard framework in formal semantics. First, as they do not seem to have truth values, it is not clear that an interpretation in terms of truth conditions is appropriate. At the same time, they show very limited ability to occur embedded in larger truth-value-bearing constructions that could help illuminate their contribution. Second, imperatives seem to guide action rather than give information, so it is not obvious how to treat them in terms of dynamic effects on belief states. It may seem more natural to associate them semantically with some sort of directive speech act. But even if we could find a non-ad hoc way of associating linguistic objects with speech acts, we would still face the problem that there is no straightforward common core to the broad range of speech acts that imperatives are used for. They easily express not only commands or requests, but also wishes, advice, curses, etc. Therefore their semantic interpretation cannot strictly determine the speech act they are used for.

In this paper we follow Schwager (2006b), who proposes to assimilate imperatives to performative modal verbs. It has been observed that modal verbs can both describe and change deontic necessities (Kamp, 1973). In the latter case, sentences containing modal verbs behave much like imperatives, for instance in that follow-ups by That's (not) true are infelicitous.

a. You may close the door (that's what the sign says).

[descriptive]

b. A: You may close the door! - B: \#That's (not) true.

[performative]

The recent literature converges on the assumption that there is no semantic difference between may as used in (11a) and in (11b) (Kamp, 1978; Schulz, 2003; Schwager, 2006b). Rather, it is under particular contextual settings that modalized sentences have a non-descriptive effect, rendering them self-verifying.

The contextual settings responsible for this effect can be characterized on the basis of the semantics for modal verbs introduced above. Schwager (2006b) singles out the following factors: (i) performatives are concerned with courses of events deemed possible by the interlocutors, hence the modal base is constituted by the context set (the set of worlds verifying all and only the mutual joint beliefs of the interlocutors, cf. Stalnaker, 1978, 2002) or a subset thereof; ${ }^{1}$ (ii) the ordering source is deontic, teleological, or bouletic ("prioritizing," cf. Portner, 2007); (iii) the

\footnotetext{
${ }^{1}$ For unembedded imperatives, the latter qualification is needed as in the case of advice the speaker typically gives information not about preferences (the odering source), but about facts in the world, (cf. Schwager, 2006b for details).
} 
speaker is taken to have perfect knowledge of both modal base and ordering source, that is, he counts as an epistemic authority on both matters; (iv) the speaker is taken to consider the prejacent possible; and $(v)$ the negation of the prejacent does not follow from what is optimal w.r.t. the speaker's wishes. ${ }^{2}$

Formally, we assume that imperatives contain a covert modal operator $\square$ similar to $⿴ 囗$ (human necessity, see Section 2.1). In contrast to modal auxiliaries, imperatives can only be used performatively. ${ }^{3}$ This falls out if the conditions $(i)-(v)$ are adopted as presuppositions introduced by $\square$. Their versatility in use (e.g., as commands, requests, wishes, or advice) follows from the context dependence of $\square$, which can take a deontic, bouletic or teleological ordering source associated with some contextually given attitude holder, typically the speaker or the addressee. For example, an imperative used for requesting can be analyzed as follows: ${ }^{4}$

Give me a call!

$\square[\mathcal{A}$ calls $\mathcal{S}$ ] is true w.r.t. $w, f, g$ iff at all worlds in $O(w, f, g) \mathcal{A}$ calls $\mathcal{S}$, where for any world $v, f(v)$ is the set of the interlocutors' mutual joint beliefs and $g(v)$ the set of propositions desired by $\mathcal{S}$ in $v$.

A use of (12) is felicitous only if it is mutual joint belief that the speaker knows what his wishes are (epistemic authority) and considers it possible for the addressee to call him. Moreover, it has to be mutual joint belief (or be accommodated as such) that the speaker does not have a strict preference against the addressee calling him.

\section{Conditional Imperatives}

With the background on conditionals and imperatives in place, we now turn to conditional imperatives. We assume that the basic assumptions we make about imperatives hold regardless of whether they occur in isolation or in conditionals. In order to arrive at a plausible analysis, then, two major questions about $\square$ need to be addressed. The first concerns its structural place in the denotations of conditional imperatives. The formal framework developed so far makes two construals available for a sentence of the form (13): ${ }^{5}$

\section{(13) If $A, B$ !}

\footnotetext{
${ }^{2}(i v)$ and (v) deviate slightly from Schwager (2006b). Instead of (iv) she requires also that the speaker be taken to consider the prejacent's negation possible. This is problematic in view of the acceptability of examples like (15b). Instead of $(v)$ she requires that the speaker is known to consider the ordering source a good guideline for acting. This formulation seems too strong as, e.g. in the case of a hearer bouletic ordering source, other propositions may be entailed s.t. the speaker does not want the hearer to act upon them.

${ }^{3}$ We follow Portner (2009) in that "performative" has to be understood in a broader sense of "nonassertive," rather than always requiring self-verification of the corresponding modalized sentence.

${ }^{4}$ The symbols $\mathcal{S}$ and $\mathcal{A}$ in the definitions refer to the speaker and the addressee, respectively.

${ }^{5}$ Strictly speaking, of course, an infinite number of construals are available, some of them (e.g., '田 $\left[\square_{[A]}[B]\right]$ ') perhaps somewhat more plausible than others (e.g., ' $\square\left[\mathbb{⿴ 囗 十}_{[A]}[B]\right]$ '). We discuss only what are generally considered the most plausible ones without justifying this selection.
} 


$$
\begin{aligned}
& \text { a. } \square_{[A]}[B] \\
& \text { b. } \mathbb{⿴ 囗 十}_{[A]}[\square B]
\end{aligned}
$$

Overt Conditional Operator (OCO)

Covert Conditional Operator (CCO)

Under the Overt Conditional Operator construal (13a), the antecedent directly restricts the modal base of the imperative operator $\square$. The Covert Conditional Operator construal (13b) locates $\square$ in the nuclear scope of an implicit epistemic operator whose modal base is restricted by the antecedent. At first glance, both analyses seem possible: On the one hand we have seen no reason why $\square$ could not be modified by the if-clause, but on the other hand a covert epistemic operator is needed independently for ordinary declarative conditionals without an overt epistemic modal, including ones with root modals in the consequent. ${ }^{6}$

The second question to be addressed concerns the modal base of $\square$. Granting that the ordering source represents the preferences of a contextually salient agent, we still need to know which set of worlds is ranked by these preferences. Here too, several possibilities are available, including for instance the speaker's belief state, i.e., the set of all worlds compatible with what the speaker believes, or the subset of antecedent-worlds within the speaker's belief state.

Although the above two questions are distinct, an answer to one cannot be evaluated without fixing an answer to the other, therefore they cannot easily be discussed separately. In the remainder of this section we explore the consequences of each of the two construals in (13) in conjunction with various plausible choices for the modal base.

\subsection{Overt Conditional Operator}

Under the OCO construal, the modal base of $\square$ is restricted by the if-clause to those worlds at which the antecedent is true. Thus for instance, (14) is true just in case the speaker's preferences rank some worlds at which the addressee sees and greets Julie higher than all worlds at which she sees Julie and does not greet her.

If you see Julie, say 'hi'!

$\square_{[\mathcal{A} \text { sees Julie }]}[\mathcal{A}$ says 'hi'] is true w.r.t. $w, f, g$ iff at all worlds in $O(w, f, g)$ at which $\mathcal{A}$ sees Julie, $\mathcal{A}$ greets her.

This interpretation is problematic. In showing this, we first discuss the closely related case of conditionals with declarative consequents containing prioritizing modals, such as If A, you should/must/have to B. Previous research has yielded conclusive arguments against an OCO construal for those sentences, but not all of those arguments carry over straightforwardly to CIs. We show that this has independent reasons and therefore does not amount to an argument for an OCO construal of CIs. We then give a novel argument against OCO which has not been made elsewhere

\footnotetext{
${ }^{6}$ Schwager (2006a) argues that it is necessary to draw on both kinds of construals in order to capture all kinds of examples. We show in passing that her arguments depend on unnecessary assumptions about the exact content of the ordering sources involved, and come up with an independent preference for an overall use of CCO construals.
} 
but applies equally to CIs and conditional declaratives with prioritizing modals in the consequent.

\subsubsection{Known problems with OCO}

It has long been known that deontic modals in declarative consequents do not seem to be modified by their antecedents. For instance, such an analysis would wrongly predict that sentences like (15a) are necessarily true (Frank, 1996; Zvolenszky, 2002). The same prediction would follow for CIs like (15b) under an OCO analysis.

a. If you drink Pepsi, you must drink Pepsi.

b. If you drink Pepsi, drink Pepsi!

Clearly once the modal base is restricted to $A$-worlds, the most preferred ones among them must again be $A$-worlds. But it is not immediately obvious for either of the examples in (15) that it is trivally true.

Not all arguments against an OCO analysis for deontic modals in conditional consequents apply to CIs. For instance, Frank (1996) notes that the interpretation of such modals can depend on the values of the ordering source at other worlds. Thus (16) may be true even at worlds at which there are laws against working longer hours and the new laws do not go through.

(16) If the new laws for opening hours of shops go through, salespeople will have to work longer.

This would be unexpected if the deontic modal operator were targeted by the antecedent, since that would merely restrict the modal base without altering the ordering source. The argument does not apply to CIs because it presupposes that the relevant ordering source can take on different values at different worlds in the modal base. While this makes sense for statements about laws, when applied to CIs it violates the condition that the speaker be an authority on the relevant preferences. ${ }^{7}$

Both for CIs and for conditionalized deontic modals, the alternative to an OCO account is a CCO account under which the antecedent restricts the modal base of a covert epistemic operator in whose nuclear scope the deontic modal is embedded. For deontic modals, Frank (1996) notes that this also seems compelling in that the covert epistemic modal postulated can be replaced with explicit modal adverbs with various forces, regardless of the force of the deontic modal:

\footnotetext{
${ }^{7}$ Schwager (2006a) comes up with an example that, at first glance, seems to violate this requirement. Assume that the speaker has a preference for law-obedience. Then, intuitively, (i) is true.

(i) If jaywalking is illegal, don't do it.

Strictly speaking, the legal status of jaywalking does not influence the speaker's preferences (as he may not know what the status is in a particular world), but it influences what the speaker's preference amounts to. This is captured correctly if the speaker's preference constantly contains the proposition ' $\lambda w$.you obey the law in $w$ '.
} 


$$
\text { If Max stays with his grandmother, he }\left\{\begin{array}{l}
\text { necessarily } \\
\text { possibly }
\end{array}\right\}\left\{\begin{array}{l}
\text { must } \\
\text { may }
\end{array}\right\} \text { walk the dog. }
$$

This argument does not apply directly to CIs because imperatives are not subject to modification with adverbs like necessarily due to their special contextual presuppositions. We can conclude from this discussion, however, that there are good arguments against $\mathrm{OCO}$ and in favor of $\mathrm{CCO}$ for deontic modals in general, and that these arguments suggest the same for CIs to the extent that they apply to them.

\subsubsection{A new argument against $O C O$}

A further problem with the OCO construal emerges when we take into account the interaction of prioritizing modality with assumptions about stereotypical courses of events. Although we discuss it only for CIs, it carries over to prioritizing modals. Consider first the sequence in (18). According to OCO, (18a) asserts that among all the worlds at which the addressee loses her job, the most preferable ones from the speaker's perspective are ones at which she accepts a lower-paying one. Notice that the speaker can continue with (18b) without contradicting himself.

(18) a. If you lose your job, take a lower-paying one.

b. But if you lose your job and have a comparable offer, don't take a lowerpaying one.

Formally, (18) is analogous to standard counterexamples to Strengthening of the Antecedent, the inference from if $A, C$ to if $A B, C$. In Kratzer-style semantics, the invalidity of this pattern is accounted for by allowing that the set of $A B$-worlds relevant for the evaluation of the latter is not contained in the set of $A$-worlds relevant for the former. As outlined in Section 2.1, the selection of the relevant worlds is driven by the ordering source. With a bouletic ordering source, Strengthening of the Antecedent fails if $A B$ is not the most preferred way for $A$ to come about and $C$ is true at the most preferred $A$-worlds but false at some or all of the most preferred $A B$-worlds.

In fact, (18) is a particularly strong counterexample in that the imperative consequents are not only contradictories but contraries of each other. In this case, the set of relevant $A B$-worlds must be disjoint from the set of relevant $A$-worlds in order for both sentences to be true. Thus in (18) it must be the case that among all the worlds at which the addressee is laid off, some of the ones at which she does not have a comparable offer are strictly preferred over all the ones at wich she does. But clearly this prediction is wrong: The CIs do not jointly imply that the speaker wishes that the addressee have no comparable offer in case she loses her job.

Intuitively, the reason why (18) lacks this implication is that losing one's job with a comparable offer in hand may be so remote or far-fetched a possibility that it does not enter the interpretation of (18a) at all. Seen this way, the selection of the antecedent-worlds relevant for the interpretation of each of the CIs appears to be driven by criteria like salience, likelihood, or stereotypicality. We do not take a stance on which of these related but distinct notions is at play in our particular 
example. For concreteness, we speak of stereotypicality in the remainder. What is important is that whichever notion is operative, it is separate from and independent of the speaker's preferences, hence the OCO analysis does not capture it.

One might attempt to remedy this problem within an OCO account by building stereotypicality into the semantics of the imperative. Whatever the details of the formal implementation of this idea might be, its semantic import would have to be that of a two-step interpretation under which the set of antecedent-worlds is first filtered by a stereotypical ordering source, and only the "best" worlds under that ranking are then further ranked according to the speaker's preferences. And if (and since) the imperative operator is to receive the same interpretation in matrix contexts as in CIs and the role of the if-clause is merely to restrict its modal base, then the modal base, restricted or not, should always be pre-ranked by stereotypicality.

Such a move would be questionable, however, for at least two reasons. One is that as we described it in the previous paragraph, the first step may well filter out as non-stereotypical all worlds in the (restricted) modal base at which the prejacent is true, rendering the imperative false. Therefore the account would predict that an imperative cannot be true unless its prejacent is stereotypical (in the case of CIs, stereotypical relative to the antecedent-worlds). Now, care must be taken in evaluating this prediction: We have to grant that speakers take into account the fact that they are about to issue the imperative and assess stereotypicality only on that basis. Thus the prediction is merely that they consider the prejacent stereotypical, given that they issue the imperative. But even this would be too strong: Mutinies and revolutions, especially failed ones, are rife with orders which take effect even though the speaker believes that the addressee will refuse to obey them. ${ }^{8}$ It may be possible to refine the double-filtering approach in such a way that epistemic uncertainty and speaker preferences interact in more subtle ways. The idea would be that the pre-selection by stereotypicality is based only on what (the speaker thinks) the most likely objective circumstances are under which the addressee must act, disregarding the question of what the addressee will stereotypically do under those circumstances. Our implementation of this refinement would rely on the notion of historical alternatives, which we introduce in Section 3.2.3 below. By then we will have collected independent arguments against the OCO, however, having to do with the second reason why the use of two ordering sources is questionable.

The second problem with the double-filtering idea arises from two theoryinternal facts. First, a dependence on the application of two ordering sources in a particular order does not generally appear to be necessary for prioritizing modals. Second, we will see in Section 3.2.2 that the approach is semantically equivalent to one version of the $\mathrm{CCO}$ account. Both run afoul of further examples which we discuss below, but only the $\mathrm{CCO}$ lends itself naturally to further refinements which

\footnotetext{
${ }^{8}$ It may seem that such a situation would violate Schwager's (2006b) Epistemic Uncertainty Constraint (EUC), which requires that the prejacent be consistent with the speaker's beliefs. But that would be a misconstrual of the EUC, which only requires that the prejacent be possible relative to the speaker's belief state. It says nothing about the prejacent's likelihood or the addressee's intentions. To satisfy the EUC, it is sufficient that the speaker is taken to believe that the addressee is (objectively) in a position to bring about the truth of the prejacent.
} 
address those problems without dramatic and stipulative departures from the standard semantics of modality. This second theory-internal argument against the OCO is ultimately decisive in our decision to favor the CCO.

\subsection{Covert Conditional Operator}

It may seem odd that one would even consider an analysis according to which imperatives are embedded under epistemic modal operators. After all, it is wellknown that imperatives resist embedding in general. But imperatives do in fact occur in a range of embedded contexts, albeit a limited one, including modal subordination (Schwager, 2006a,b) and reported speech (Crnič and Trinh, 2009; Portner, 2007; Schwager, 2006b; Poschmann and Schwager, 2008). Such facts undermine the claim that imperatives cannot occur in embedded contexts and make a CCO analysis worth exploring.

Under a $\mathrm{CCO}$ construal, If $A, B$ ! states that at those worlds at which $A$ is true, $B$ is preferred. Thus the conditional consequent is evaluated point-wise at the antecedent worlds. If the force of the outer epistemic modal is human necessity relative to a stereotypical ordering source, then the truth conditions are as in (19).

If you get lost, call me!

a. $\mathbb{⿴ 囗 十 ~}_{\text {[you get lost] }}\left[\right.$ [you call me] is true w.r.t. $w, f, g$ iff for all worlds $w^{\prime} \in$ $O(w, f, g)$ at which $\mathcal{A}$ gets lost, $\square\left[\right.$ you call me] is true at $w^{\prime}, f^{\prime}, g^{\prime}$.

b. $\square\left[\right.$ you call me] is true w.r.t. $w^{\prime}, f^{\prime}, g^{\prime}$ iff at all worlds in $O\left(w^{\prime}, f^{\prime}, g^{\prime}\right), \mathcal{A}$ calls $\mathcal{S}$.

This analysis assumes that two modal operators are involved in CIs, each with a modal base and an ordering source, referred to in (19) as $f, g$ and $f^{\prime}, g^{\prime}$, respectively. By assumption, the modal base of the outer epistemic operator is the speaker's belief state, ranked by a stereotypical ordering source. We also assume, based on the meaning of imperatives in general, that the ordering source of the embedded imperative reflects the contextually given preferences. But what should the modal base of the embedded imperative be?

Imperatives in CI consequents, like matrix imperatives, are concerned with possible courses of events. Thus (19) should not be falsified by the existence of worlds where calling is superfluous because the addressee miraculously develops perfect knowledge of the area the moment she is lost - such worlds may be highly desirable but have no effect on the interpretation of the imperative in the consequent, just as they are disregarded by stand-alone imperatives. This is ensured by considering only worlds in the context set. In the following, we discuss three possibilities in turn: the context set itself, the antecedent worlds within the context set, and the set of historical alternatives of each world in the context set. The discussion thus proceeds from the most "gobal" to the most "local" notion. We show that the last option is most adequate in light of certain basic intuitions about CIs. 


\subsubsection{Context set}

First, suppose the modal base relevant for the imperative consequent is the set of all worlds in the context set, and consider the CI in (20).

If you get lost, call me!

true at $w$ iff for all stereotypical worlds $w^{\prime}$ consistent with $\mathcal{S}$ 's beliefs in $w$ such that $\mathcal{A}$ gets lost at $w^{\prime}$, the preferences at $w^{\prime}$ rank highest those worlds in the context set at which $\mathcal{A}$ calls $\mathcal{S}$.

As mentioned above, we follow Stalnaker $(1978,2002)$ in taking the context set to verify exactly the beliefs which the interlocutors implicitly agree to take for granted for the sake of the conversation. The most relevant feature of this construct is that it represents not only their shared beliefs about the external facts, but also their mutual beliefs about each other. For instance, it may be mutually believed for some proposition $p$ that the speaker does and the addressee does not have a definite belief as to whether $p$ is true or false. In such a case, at each world $w^{\prime}$ in the context set, $p$ is either true at all worlds in the speaker's belief state at $w^{\prime}$ or false at all of them, while the addressee's belief state at $w^{\prime}$ comprises both $p$-worlds and $\bar{p}$-worlds.

Suppose for example that the relevant preferences are the speaker's, who (i) has an unconditional preference for the addressee not to get lost; and (ii) has no unconditional preference for or against being called. Intuitively the CI in (20) is consistent with this situation. But according to the truth conditions, it is false. For consider a stereotypical world $w^{\prime}$ in the speaker's belief state at which the antecedent is true, i.e., the addressee gets lost. By the authority condition and assumption $(i)$, the speaker's preferences at $w^{\prime}$ rank highest those worlds in the context set at which the addressee does not get lost. By assumption (ii), that set of highestranking worlds contains both worlds at which she calls him and worlds at which she does not, hence the CI is false. Intuitively, if the CI is true, then it is because among the worlds at which the addressee gets lost, the speaker ranks highest those at which she calls. But assumption ( $i$ ) implies that those worlds, like all antecedentworlds, are strictly outranked by ones at which she does not get lost.

Thus if the entire context set is taken to be the modal base for the imperative, then (20) implies that an unconditional preference for calling be in effect at the relevant antecedent-worlds. On the other hand, the CI does not imply that an unconditional preference for calling is in effect at all worlds in the speaker's belief state. These requirements cannot both be met unless either the modal base or the ordering source (or both) is allowed to vary between worlds depending on the truth or falsehood of the antecedent. We maintain that the ordering source should not vary: Even if the authority condition were not as eminently plausible a condition in itself as it is in our view, it would still be questionable to make the truth of the CI dependent upon uncertainty on the part of the speaker about the relevant preferences.

The upshot is that the relevant modal base for examples like ours must be a proper subset of the context set which contains only worlds at which the addressee is lost. What should this subset be? 


\subsubsection{Antecedent Worlds ${ }^{9}$}

One possibility is to make the set of antecedent-worlds in the context set the modal base of the imperative consequent. In our example, repeated as (21) with adjusted truth conditions, this means that at worlds at which the addressee gets lost, only worlds at which she gets lost are relevant to the truth of the consequent; similarly for worlds at which she does not get lost. ${ }^{10}$

If you get lost, call me!

true in $w$ iff for all stereotypical worlds $w^{\prime}$ consistent with $\mathcal{S}$ 's beliefs in $w$ such that $\mathcal{A}$ gets lost at $w^{\prime}, \mathcal{S}$ 's preferences at $w^{\prime}$ rank highest among the worlds in the context set at which $\mathcal{A}$ gets lost, those at which $\mathcal{A}$ calls $\mathcal{S}$.

Suppose again that the speaker prefers that the addressee not get lost, and consider a world $w^{\prime}$ in the speaker's belief state at which she does get lost. What should we assume about the speaker's preferences in order to derive plausible truth conditions for the CI?

The first thing to note is that the speaker's desires should not directly determine a preference for calling. This follows again from the assumption that the speaker knows his own preferences (i.e., that they are the same at all worlds in his belief state) together with the observation that no preference for calling is implied by (21) unless the addressee is in fact lost. Nor is it sufficient for the speaker's preferences to be limited to an attitude towards the truth or falsehood of the antecedent. For suppose, as in the last subsection, that the ordering source at $w^{\prime}$ contains only the proposition that the addressee not get lost. Since the modal base is restricted to worlds at which the addressee is lost, this ordering source would not differentiate between them, and the order it induces would be the universal relation on the modal base. But then (21) is false, unless it is already known that the addressee calls at all worlds at which she lost, which is clearly not implied.

So we need to assume that the speaker's preferences jointly with the modal base yield a preference for calling. This means that among the antecedent-worlds in the context set, his ordering source ranks some higher than others. For instance, the speaker may want to meet with the addressee for dinner, and among all the worlds at which she is lost, the ones at which the dinner takes place as hoped are ones at which she calls. No global preference for calling is implied, since it may well be that not getting lost alone is also sufficient to secure the dinner together. Crucial for getting plausible truth conditions for CIs in this way is that the ordering source include propositions which "cut across" the set of antecedent worlds, ranking some higher than others.

Thus by restricting the modal base to the antecedent-worlds in the context set, we can account for the truth of (21) in the given scenario. This is an improve-

\footnotetext{
${ }^{9}$ Schwager (2006a) considers only this possibility, but dismisses it as ad hoc. She therefore considers examples like (20) evidence in favor of an OCO construal. We argue below that it is not OCO, but a certain modification for $\mathrm{CCO}$, that makes the best predictions even for these cases.

${ }^{10}$ This analysis, as well as the one in Section 3.2.3 below, predict that the "Pepsi CI" in (15b) is trivially true. We reserve an a discussion of this prediction for future work. XXXXXXXXXX
} 
ment over the interpretation in Section 3.2.1 above, which took the entire context set as the modal base. Nevertheless, having this in place, the account still runs into problems in connection with different types of contextually possible antecedent worlds. For example, consider the following variant of the scenario underlying (21). The addressee will spend her day sightseeing either in Chicago or in Evanston. The speaker's preferences are encoded in an ordering source which, at each world in the speaker's belief state, contains two propositions: $(i)$ that the speaker and the addressee meet for dinner at the end of the day; and (ii) that they meet for dinner and save money. Among the stereotypical worlds in the context set at which the addressee gets lost, we find both worlds where she gets lost in Evanston and worlds where she gets lost in Chicago, but no matter where she gets lost, the only way to secure the dinner is to get in touch. Calling is an option in either city, but it is also costly. In Evanston, but not in Chicago, free wireless internet is a cost-saving alternative.

Now the set of worlds at which the antecedent is true includes ones in which she is lost in Chicago and ones in which she is lost in Evanston. Clearly most preferred among these, given the preferences $(i)$ and (ii) above, are ones at which she gets in touch via free wireless internet, for these are worlds at which she saves money while also securing the joint dinner. In this scenario, the formal account makes two false predictions: First, (21) now comes out false, even though intuitively calling is still the best course of action for the addressee. Secondly, we predict that the CI in (22) is true. But intuitively (22) should come out false: The addressee may well get lost in Chicago, and in that case emailing is not even an option!

(22) If you get lost, email me!

This example highlights a problem which we have sidestepped so far, but which must be addressed eventually if we are to obtain a plausible account of CIs: By using the entire set of contextually plausible antecedent-worlds as the modal base for the imperative we are missing the fact that the question of what the best course of action is - both in the sense of which actions are available to the addressee and in terms of the likely consequences of those actions - may depend on contingencies that are not mentioned in either constituent of the CI. In our scenario, the speaker's preferences are responsible for the truth of certain conditional nonimperatives, such as the sentences in (23), but not the CI in (22).

a. If you get lost, I hope it is in Evanston.

b. If you get lost, it would be good if you could email me.

The crucial difference is, of course, that while the speaker may hope that the addressee is lost in Evanston and not in Chicago, he cannot tell/ask her to be lost in Evanston. The Evanston-worlds are irrelevant to the interpretation of the imperative at worlds at which the addressee is lost in Chicago, simply because at those worlds it is beyond her powers to make it such that she is lost anywhere else. 


\subsubsection{Historical Alternatives}

The conclusion of the last subsection suggests that objective circumstances should play a role in the interpretation of imperatives. The third option for determining the modal base rests on the idea that facts which are unalterable at the world and time of evaluation must be accounted for. The formal implementation of this idea requires that we add a temporal dimension to the model. Here we only give an informal description of the main points.

The basic idea is to restrict the modal base, at each world $w^{\prime}$ in the speaker's belief state, to the historical alternatives of $w^{\prime}$ at the time at which the antecedent is evaluated. The notion of a historical alternative has been found to play a role in the semantic interaction between expressions of modality, including conditionals, and temporality (see Thomason, 1984 for a discussion of the formal background, and Condoravdi, 2002, Kaufmann, 2005 for linguistic applications). It is meant to account for the intuition that there is a fundamental asymmetry between a "fixed" past and present on the one hand, and an "open" future, on the other: At any time $t$, each possible world has exactly one history leading up to and including $t$, whereas there are multiple possible continuations from $t$ on; which of these possible courses of events is the actual one is not decided until the relevant later time comes to pass. Formally, for any world $w$ and time $t$, the set of historical alternatives of $w$ at $t$ consists entirely of worlds indistinguishable from $w$ at all times up to $t$. Variation among the historical alternatives is only possible with respect to times later than $t$.

Among the linguistic facts whose analysis appeals to this asymmetry is that modal sentences with past or present reference in the prejacent can only have an epistemic interpretation, reflecting subjective uncertainty, whereas with future reference an additional, metaphysical or objective reading becomes available. But objective uncertainty has epistemic consequences: What is not objectively settled cannot possibly be known in advance. Formally, this amounts to a constraint on the relation between the historical alternatives at a given time and the epistemic alternatives modeling the speaker's belief state at that time: Epistemic states must be unions of entire classes of historical alternatives; they must not "cut across" them, including some but not all historical alternatives of some world at the given time. Such cross-cutting would amount to knowledge of future facts which are not yet objectively determined, which we assume is impossible. Crucially for our purposes, for any time $t$ and world $w^{\prime}$ in the speaker's belief state at $t$, the set of historical alternatives of $w^{\prime}$ at $t$ is guaranteed to be a subset of the speaker's belief state at $t$.

Now, the interpretation of CIs, and indicative conditionals in general, in a model which takes this interaction between modality and time seriously, is complicated by the fact that the antecedent may be evaluated at times later than the speech time. This is the case in (21) and (22) above: The antecedent can be paraphrased as If (and when) you get lost at some (non-past) time .... Intuitively, the courses of action available to the addressee in case she does get lost at some future time are determined at that future time, not at the speech time at which the $\mathrm{CI}$ is issued and interpreted. This is predicted as a consequence of a temporal shift into the future that is always avaiable with indicative conditionals (Kaufmann, 2005) and which, 
importantly, affects the interpretation of both the antecedent and the consequent. Thus if historical alternatives determine the modal base relevant for an imperative consequent, it must be kept in mind that those are the historical alternatives at its (possibly forward-shifted) interpretation time, not necessarily those at speech time.

With this background in place, let us now see how the restriction of the modal base to the historical alternative fares with respect to our example (21), repeated here as (24).

If you get lost, call me!

true in $w$ iff for all stereotypical worlds $w^{\prime}$ consistent with $\mathcal{S}$ 's beliefs such that $\mathcal{A}$ gets lost at $w^{\prime}, \mathcal{S}$ 's preferences rank highest among the historical alternatives of $w^{\prime}$ those at which $\mathcal{A}$ calls $\mathcal{S}$.

The first thing to note that these truth conditions are stronger than those in (21) above, where the imperative was evaluated relative to the whole set of (relevant) antecedent-worlds. Under that interpretation the imperative was true at all antecedent-worlds if (and only if) it was true at any of them. This is because the modal base is the same at all relevant antecedent-worlds (namely the set of antecedent-worlds), and the ordering source is constant by assumption. In (24), in contrast, the set of antecedent-worlds is partitioned into classes of historical alternatives. Suppose the CI was true under the former interpretation. This means that the addressee calls at all of the most preferred ones among the antecedent-worlds. Each of them will also be among the most preferred ones within its local class of historical alternatives - but, crucially, not vice versa: There may be worlds that are most preferred locally (among their historical alternatives) but not relative to the entire set of antecedent worlds. This means that a CI that was true under the interpretion in (21) may now be false under (24). On the other hand, the increased locality cannot in itself render a CI that was false relative to the set of antecedent-worlds true relative to historical alternatives.

This argument makes clear that so far, we have only solved part of the problem. Recall that in Section 3.2.2 we showed that by taking the antecedentworlds as the modal base we ended up with two incorrect predictions for the Chicago/Evanston scenario: Sentence (24) was predicted to be false, while (22), repeated here as (25), was predicted to be true.

(25) If you get lost, email me!

Now the analysis in terms of historical alternatives correctly predicts the falsehood of (25). Emailing is not even an option at some of the (relevant) antecedent-worlds, let alone a preferred one. And since the CI requires for its truth that the consequent come out true point-wise at all (relevant) antecedent-worlds, the CI is correctly predicted to be false.

\subsubsection{Metalinguistic considerations}

While the falsehood (25) is now accounted for, (24) is still not predicted to be true in the Chicago/Evanston example. The problem is that our truth conditions require 
that in order for the CI to be true, calling must be the preferred course of action at all (relevant) antecedent-worlds. This is not the case in the scenario: At worlds at which the addressee gets lost in Evanston and therefore has access to email, the worlds ranked highest by the speaker's preferences do not include any calling worlds, as they are too costly.

Let us be clear about the scope of this problem. Although it turned up in our discussion of conditional imperatives, it is in fact not confined to this particular set of data. It arises just the same if we restrict our attention to simple imperatives like (26) relative to a scenario which is similar to the Chicago/Evanston story discussed thus far, except that the addressee is lost already, talking to the speaker on the phone, and the only way to ensure their joint dinner is to get in touch with Eva (subject to the same contingencies w.r.t. money and wifi availabilities). If the speaker does not know in which city the addressee is and the facts are as before, then (26) is felt to be true. Again the problem is not confined to imperatives but arises with modal verbs just the same. ${ }^{11}$
a. Call Eva!
b. You should call Eva!

The general problem can be stated as follows: If the speaker wants to give advice as to what is the best course of action but does not know what the circumstances are or will be under which the addressee has to act, he can apparently call an action $\alpha$ the best option even if, strictly speaking, $\alpha$ is not the best option at all relevant worlds, but dominated "locally" at some worlds by an option $\beta$. Note that this is subject to two constraints: $\alpha$ must be available at all relevant worlds, and there are relevant worlds at which $\beta$ is not available (or possibly, in alternative scenarios, worlds at which $\beta$ is strongly dispreferred). This seems to be at odds with truth conditions that flatly state that $\alpha$ is "the best option." One possible adjustment would be to weaken the truth conditions to require that $\alpha$ be "the best option guaranteed to be available." We might consider tying this to the epistemic uncertainty condition imposed on imperatives in general, which requires mutual joint belief that the speaker considers the prejacent of the imperative possible. Alternatively, we could require that $\alpha$ be "optimal"according to some weighted global comparisons scheme. But we won't pursue these directions here. Rather, we want to step back and reconsider the felicity of the sentence in the given scenario. Strictly speaking, shouldn't the speaker have said something along the lines of (27)?

(27) If you get lost, call me or email me, depending on whether you are in Chicago or in Evanston.

(27) would certainly have been an alternative, yet (24) seems acceptable, too. Let us revisit the description of the scenario: We assumed an overall goal of meeting for

\footnotetext{
${ }^{11}$ The status of examples involving must or have to may be less clear, though, in this scenario. This is in line with observations in the literature on anankastic conditionals (e.g. von Stechow et al., 2005), where it is also argued that should and ought to are more readily felt to be true (and imperatives pattern with them, cf. Schwager, 2006b) than must and have to.
} 
dinner, and a subsidary goal of saving money if possible. But if these were his only concerns, the speaker should indeed have uttered (27), as (24) may well not lead to the best outcome for him. This, we contend, is the correct judgment, whereas the impression that (24) is true is due to tacit additional preferences, presumably either (a) that the addressee have a simple enough plan, or $(b)$ that the speaker not have to give lengthy explanations. Both would be good reasons to prefer (24) over (27).

Yet, $(a)$ and $(b)$ do not just account for the speaker's choice of words, they also interact with the truth conditions (which crucially rely on speaker's preferences) and render (24) true when added to the ordering source for the CI. Note that $(a)$ is unproblematic: We can ensure that having a simple plan outranks saving money by construing the preferences as $(i)$ we meet for dinner, (ii) we meet for dinner and you follow a simple plan, (iii) we meet for dinner and you follow a simple plan and we save money. In that case, the highest ranked worlds are worlds at which speaker and addressee meet for dinner and the addressee follows the simple plan of calling (which is the best simple plan in view of the third goal of saving money, outranking for example options like calling a helicopter). Things are less straightforward with $(b)$, which requires an adequate formalization of the interaction between meta-linguistic (roughly, Gricean) and extra-linguistic preferences. The issue is clearly not confined to CIs or imperatives.

Note that these more fine-grained preferences only help if the ordering happens pointwise. The solution does not carry over to the solution in terms of antecedent worlds or its equivalent in terms of an OCO sensitive to stereotypicality. Those theories still predict (22) to be true and (19) to be false.

\section{Conclusions}

We have shown that conditional imperatives of the hypothetical subtype can be given a promising analysis if the imperative is understood as a modal operator of human necessity embedded under a covert epistemic modal which is restricted to quantify over antecedent worlds. We have discussed various possiblities of what the imperative modal operator itself could quantify over. Ultimately, we believe to have shown that pointwise quantification over (stereotypical) alternatives to each of the worlds considered by the epistemic modal operator makes the best predictions. Still, the discussion at the end of Section 3.2.4 shows that ordering sources seem to be a somewhat crude tool for modeling the ranking of preferences, let alone the interaction between extra-linguistic and meta-linguistic preferences. This could be taken as an argument to switch to a framework that deals with utilities directly. Lastly, we would like to point out that the remaining worries do not apply exclusively to conditional imperatives, but affect conditionals containing other non-epistemic modals as well. We consider this further support for theories that assimilate modals and imperatives. 


\section{References}

Bhatt, Rajesh and Roumyana Pancheva: 2006, 'Conditionals', in M. Everaert, H. van Riemsdijk, R. Goedemans, and B. Hollebrandse (eds.), The Blackwell Companion to Syntax, Vol. 1, 638-687. Wiley-Blackwell.

Condoravdi, Cleo: 2002, 'Temporal Interpretation of Modals: Modals for the Present and for the Past', in D. I. Beaver, L. Casillas, B. Clark, and S. Kaufmann (eds.), The Construction of Meaning, 59-88. CSLI Publications.

Crnič, Luka and Tue Trinh: 2009, 'Embedding Imperatives in English', in A. Riester and T. Solstad (eds.), Proceedings of Sinn und Bedeutung 13, 113127.

Frank, Anette: 1996, Context Dependence in Modal Constructions, Doctoral Dissertation, Institut für maschinelle Sprachverarbeitung (Stuttgart).

Kamp, Hans: 1973, 'Free Choice Permission', Proceedings of the Aristotelian Society 74, 57-74.

Kamp, Hans: 1978, 'Semantics versus Pragmatics', in F. Guenthner and S. J. Schmidt (eds.), Formal Semantics and Pragmatics for Natural Languages, 255-287. Reidel, Dordrecht.

Kaufmann, Stefan: 2005, 'Conditional Truth and Future Reference', Journal of Semantics 22, 231-280.

Kratzer, Angelika: 1981, 'The Notional Category of Modality', in J. Eikmeyer and H. Riesner (eds.), Words, Worlds, and Contexts, 38-74. Walter de Gruyter.

Portner, Paul: 2007, 'Imperatives and modals', Natural Language Semantics 15, 351-383.

Portner, Paul: 2009, Modality. Oxford University Press.

Poschmann, Claudia and Magdalena Schwager: 2008, 'Shifted imperatives and echoquestions'. Ms., University of Frankfurt/University of Göttingen.

Schulz, Katrin: 2003, 'You may read it now or later: A Case Study on the Paradox of Free Choice Permission'. Master Thesis University of Amsterdam.

Schwager, Magdalena: 2006a, 'Conditionalized Imperatives', in C. Tancredi, M. Kanazawa, I. Imani, and K. Kusumoto (eds.), Proceedings of SALT XVI.

Schwager, Magdalena: 2006b, Interpreting Imperatives, Doctoral Dissertation, University of Frankfurt/Main.

Stalnaker, Robert: 1978, 'Assertion', in P. Cole (ed.), Syntax and Semantics 9, 315332. Academic Press, New York.

Stalnaker, Robert: 2002, 'Common Ground', Linguistics and Philosophy 25, 701721.

von Stechow, Arnim, Sveta Krasikova, and Doris Penka: 2005, 'Anankastic Conditionals'. Ms., Universität Tübingen.

Thomason, Richmond H.: 1984, 'Combinations of Tense and Modality', in D. Gabbay and Günthner (eds.), Handbook of Philosophical Logic, 2 edn, 205234.

Zvolenszky, Zsófia: 2002, 'Is a Possible-Worlds Semantics of Modality Possible? A Problem for Kratzer's Semantics', in B. Jackson (ed.), Proceedings of SALT XII. 